\title{
Stromal protein periostin identified as a progression associated and prognostic biomarker in glioma via inducing an invasive and proliferative phenotype
}

\author{
HONGJUN WANG $^{1 *}$, YONGZHI WANG ${ }^{2 *}$ and CHUANLU JIANG ${ }^{1}$ \\ ${ }^{1}$ Department of Neurosurgery, The Second Affiliated Hospital of Harbin Medical University, Harbin; \\ ${ }^{2}$ Department of Neurosurgery, Tiantan Hospital, Capital Medical University, Beijing, P.R. China
}

Received November 28, 2012; Accepted January 24, 2013

DOI: $10.3892 /$ ijo.2013.1847

\begin{abstract}
To explore the expression pattern, prognostic value and functional role of stromal periostin (POSTN) in glioma patients, POSTN expression was measured using the Agilent Whole Human Genome Oligo Microarray in 220 frozen glioma tissues. We analyzed POSTN expression in 71 independent validated glioma samples using immunohistochemistry. The expression levels of POSTN were relative to glioma grade progression and inversely correlated with overall survival in high-grade glioma patients (anaplastic gliomas and glioblastomas). Gene ontology (GO) analysis performed using DAVID showed that the gene sets related to cell migration and proliferation were significantly enriched in the cases with POSTN overexpression. Functional analyses in LN229 and U87 cells revealed that POSTN was involved in cell invasion and proliferation. MMP-9 was an effector of POSTN signaling in glioma cells. The expression of stromal protein POSTN is relative to glioma grade progression and confers a poor prognosis via promoting cellular invasion and proliferation in high-grade glioma patients.
\end{abstract}

\section{Introduction}

Glioma is the most common primary brain tumor, but its prognosis has changed little over the past 30 years, despite comprehensive and intensive treatment. The invasive growth pattern of glioma cells is partially responsible for its poor response to treatment. A major pathophysiological feature of

Correspondence to: Professor Chuanlu Jiang, Department of Neurosurgery, The Second Affiliated Hospital of Harbin Medical University, 246 Xuefu Road, Harbin, Heilong Jiang Province 150086, P.R. China

E-mail: jc16688@163.com

${ }^{*}$ Contributed equally

Key words: malignant glioma, invasion, survival, periostin, stromal protein, proliferation gliomas is their ability to invade diffusely into the surrounding brain tissues $(1,2)$, even across the brain lobes, producing new growth foci and finally spreading throughout the central nervous system. Limiting the invasive ability of gliomas thus represents a potential approach for curing patients and prolonging survival.

Growing evidence has implicated the secreted stromal protein periostin (POSTN) in many tumors (3-6), including breast cancer, prostate cancer and cholangiocarcinoma (7). POSTN has been confirmed to play roles in invasion/metastasis $(8,9)$, cell proliferation $(10,11)$, migration $(12)$ and angiogenesis $(9,13)$, but its function and mechanism in all grades of glioma is not completely clear, although there are some relative reports in glioblastoma (14-16). Here, we report the expression pattern of POSTN using the expression values from microarrays of 220 frozen glioma tissues and investigated the expression of POSTN in relation to glioma grade progression validated by 71 glioma samples using immunohistochemistry. In addition, to underscore the potential biological insights of POSTN 89 GBM samples were subjected to whole genome gene profiling. Integrated analysis of POSTN and whole genome gene expression patterns showed that overexpression of POSTN was tightly correlated with the gene sets related to cell invasion and proliferation. Functional assays showed that POSTN might serve as a potential target for anti-invasion and anti-proliferation therapies in glioma patients.

\section{Materials and methods}

Tissue samples. Two hundred and twenty frozen glioma samples were obtained from newly diagnosed patients treated by the CGGA Group. Tumor histology of all patients was confirmed independently by two neuropathologists based on the 2007 edition of WHO classification of central nervous system tumors. All the patients in the study received similar treatments. The inclusion criteria were as follows: i) The patients were treated with conventional therapies consisting of maximal surgical resection, followed by radiotherapy and/or chemotherapy. ii) Patients who received radiotherapy or chemotherapy before admission or died from non-glioma-related diseases were excluded from this study. iii) All the patients had follow-up and age $>18$ years. This study was approved by 
the Research Ethics Committee of Beijing Tiantan Hospital. Written informed consent was obtained from each patient.

RNA extraction and microarray analysis. All the tissue samples were immediately snap-frozen in liquid nitrogen after surgery. A frozen section from each tumor was stained with hematoxylin and eosin for assessment of the percentage of tumor cells before RNA extraction. Only samples with $>80 \%$ tumor cells were selected. Total RNA from frozen tumor tissues was extracted from frozen tumor tissues by using a Total RNA Isolation kit (Ambion, Austin, TX) according to the manufacturer's protocol. RNA concentrations were measured using a NanoDrop ND-1000 spectrophotometer (NanoDrop Technologies, Houston, TX). The expression profiling of all the samples was tested with the Agilent Whole Human Genome Oligo Microarray, which is in a $4 x 44 \mathrm{k}$ slide format: each block represents $>41,000$ probesets. All the steps of sample-labeling, hybridization, washing and scanning steps were conducted at the laboratory in the Cancer Institute/ Hospital of Peking Union Medical College, following the manufacturer's instructions. In brief, POSTN-labeled cRNA was generated from $500 \mathrm{ng}$ input total RNA by in vitro transcription using Agilent's Low RNA Input Linear Amplification kit Plus and $1.65 \mu \mathrm{g}$ cRNA from each labeling reaction was hybridized to one block of the microarray. After hybridization, slides were washed and then scanned with the Agilent G2565BA Microarray Scanner System. Fluorescence intensities on scanned images were extracted and preprocessed by Agilent Feature Extraction Software (v9.1). Data normalization and filtering was performed using the GeneSpring GX 11.0 (Agilent Technologies). Only those genes with expression levels marked as present or marginal in all of the chips (blocks) passed through the quality filtering. Then, measurements were set to $<0.01$ and the chip data were normalized to the 50 th percentile of the intensity of each chip. Significant analysis of microarrays (SAM) was implemented on low-grade glioma (astrocytoma, oligodendroglioma and oligoastrocytoma) and high-grade glioma (anaplastic gliomas and glioblastomas). Genes that were significantly up- or downregulated were identified using SAM (17). SAM assigns a score to each gene on the basis of a change in gene expression relative to the standard deviation of repeated measurements. Analysis parameters (Delta) were set to result in false discovery rate (FDR) $<0.2$.

Gene ontology analysis. To obtain more information on the biologic processes related to POSTN expression in 89 glioblastomas, we performed gene-expression profiling using Agilent Whole Human Genome Array and Gene ontology analysis (GO) (david.abcc.ncifcrf.gov) (Table I).

Validated samples and treatment. Surgical specimens of 71 glioma patients included 24 astrocytoma (WHO grade II), 9 anaplastic astrocytoma (AA, WHO grade III) and 38 glioblastomas (WHO grade IV) were included into this retrospective study at the Glioma Center of Beijing Tiantan Hospital. The inclusion criteria were the same as for microarray samples. Progression-free survival (PFS) was defined as the time from surgical resection to the first MRI-confirmed recurrence or until death. Overall survival (OS) was defined as the period of time from surgical resection to death. The glioma tissues and data were provided by China Glioma Genome Atlas (CGGA, www.CGGA.org.cn). Standard treatment consisted of surgical resection and postoperative radiotherapy, with adjuvant chemotherapy. The extent of resection was assessed using postoperative enhanced MRI within $72 \mathrm{~h}$ and graded as total or subtotal resection. The patients received standard radiotherapy (60 Gy in 2-Gy fractions, with five fractions administered per week) of the contrast-enhancing lesion (plus a 2-cm safety margin and the area of preoperative edema). This was approved by the Ethics Committee of Beijing Tiantan Hospital and was based on the criteria of the Helsinki convention. Written informed consents was provided by each participant.

Immunohistochemistry. Paraffin-embedded specimens were cut into $4-\mu \mathrm{m}$ sections and baked at $65^{\circ} \mathrm{C}$ for $30 \mathrm{~min}$. The sections were deparaffinized with xylene and rehydrated. Sections were submerged into EDTA ( $\mathrm{pH}$ 8.0) and autoclaved for antigen retrieval, then treated with $3 \%$ hydrogen peroxide, followed by incubation with $1 \%$ FBS. Anti-POSTN antibody (ab14041, rabbit polyclonal, USA, 1:200 dilutions) was added and incubated overnight at $4^{\circ} \mathrm{C}$. For negative controls, the primary antibody was replaced by normal mouse serum. Horseradish peroxidase (HRP) labeled secondary antibody in the MaxVision ${ }^{\mathrm{TM}}$ HRP-Polymer anti-rabbit IHC kit (KIT-5930 Maixin Biol, Fu Zhou, China) was applied and incubated for $30 \mathrm{~min}$ at room temperature, followed by $5-\mathrm{min}$ incubation at room temperature with $\mathrm{DAB}$ provided in the kit for color development. The sections were finally counterstained with haematoxylin and mounted with Permount (BIOS, Beijing, China). Results were visualized and photographed under a light microscope (Olympus BX-51; Olympus Optical). The degree of immunostaining of sections was viewed and scored separately by two independent investigators. The scores were determined by combining the proportion of positively stained tumor cells and the intensity of staining. Scores from the two investigators were averaged for further comparative evaluation of the POSTN expression. The intensity of staining was recorded on a scale of 0 (no staining), 1 (weak staining, light yellow), 2 (moderate staining, yellowish brown) and 3 (strong staining, brown).

Cell lines and cell culture. Human glioblastoma cell lines U87 and LN229 were obtained from the Institute of Biochemistry and Cell Biology, Chinese Academy of Science, Shanghai, China. The cells were maintained in Dulbecco's modified Eagle's medium (DMEM; Gibco, USA) supplemented with $10 \%$ fetal bovine serum and incubated at $37^{\circ} \mathrm{C}$ in $5 \% \mathrm{CO}_{2}$. Upon $80 \%$ confluency, cells were starved in DMEM with $1 \%$ FBS for $24 \mathrm{~h}$ and maintained in this low serum condition for the course of treatment.

POSTN gene knockdown by siRNA. Specific oligos (21-bp) targeting the POSTN gene were selected (Shanghai GenePharma Co., Ltd.). SiRNA2, the most efficiency was screened to knock down POSTN from three siRNAs (Table II). Logarithmically growing cells were seeded at a density of $10^{5}$ cells per $6-\mathrm{cm}$ dish and transfected with $5 \mu \mathrm{mol}$ POSTN siRNA using Lipofectamine 2000 (Invitrogen) according to the manufacturer's instructions. Forty-eight hours later, cells were used for in vitro functional assay as described below. 
Table I. Gene sets enriched in glioblastoma samples with POSTN overexpression.

\begin{tabular}{|c|c|c|c|c|}
\hline Name & Count & Fold enrichment & p-value & FDR \\
\hline GO:0006928-cell motion & 94 & 2.00083707 & $6.19 \mathrm{E}-11$ & $1.14 \mathrm{E}-07$ \\
\hline GO:0051270-regulation of cell motion & 49 & 2.56694316 & $1.13 \mathrm{E}-09$ & $2.10 \mathrm{E}-06$ \\
\hline GO:0016477-cell migration & 60 & 3.444752445 & $8.02 \mathrm{E}-09$ & $1.16 \mathrm{E}-05$ \\
\hline GO:0042127-regulation of cell proliferation & 128 & 1.64441988 & $1.11 \mathrm{E}-08$ & $2.05 \mathrm{E}-05$ \\
\hline GO:0043122-regulation of I- $\kappa$ B kinase/NF- $\kappa$ B cascade & 31 & 2.929242977 & 8.69E-08 & $1.61 \mathrm{E}-04$ \\
\hline GO:0030334-regulation of cell migration & 41 & 2.452870574 & $1.18 \mathrm{E}-07$ & 2.17E-04 \\
\hline GO:0048870-cell motility & 61 & 2.008949134 & $1.75 \mathrm{E}-07$ & $3.24 \mathrm{E}-04$ \\
\hline GO:0008284-positive regulation of cell proliferation & 74 & 1.807210993 & $6.04 \mathrm{E}-07$ & 0.001116781 \\
\hline GO:0007249-I- $\kappa$ B kinase/NF- $\kappa$ B cascade & 21 & 3.370204285 & $1.45 \mathrm{E}-06$ & 0.002671136 \\
\hline GO:0008283-cell proliferation & 69 & 1.600074053 & $9.30 \mathrm{E}-05$ & 0.171734749 \\
\hline
\end{tabular}

Table II. SiRNA and negative control strand.

\begin{tabular}{lll}
\hline siRNA1 & GCCAUCACAUCGGACAUAUTT & AUAUGUCCGAUGUGAUGGCTT \\
siRNA2 & GGUCCUAAUUCCUGAUUCUTT & AGAAUCAGGAAUUAGGACCTT \\
siRNA3 & GCCCUGGUUAUAUGAGAAUTT & AUUCUCAUAUAACCAGGGCTT \\
Negative control & UUCUCCGAACGUGUCACGUTT & ACGUGACACGUUCGGAGAATT \\
\hline
\end{tabular}

SiRNA strand was from $5^{\prime}$ to $3^{\prime}$.

Transwell invasion assay. The transwell invasion assay was done in 24-well cell culture chambers using transwell inserts (Corning Life Sciences, NY, USA) with $8-\mu$ m pore membrane precoated with Matrigel (BD Bioscience, San Jose, CA, USA). U87 and LN229 cells were plated at the density of $1 \times 10^{4}$ per upper well in $200 \mu \mathrm{l}$ culture medium (DMEM, no FBS), control group, siRNA control group and siRNA group especially. The lower chamber was filled with $500 \mu 1$ medium (DMEM, 12\% FBS). The cells were allowed to invade for $24 \mathrm{~h}$, after which, the non-invading cells with Matrigel matrix were removed from the upper surface of the membrane by scrubbing with a cotton-tipped swab. Cells on the lower surface of the filter were fixed for $30 \mathrm{~min}$ in methanol and glacial acetic acid mixture (3:1), air-dried briefly and stained with crystal violet. The mean number of invaded cells was counted from five preselected microscopic fields at magnification $\mathrm{x} 200$, all experiments were performed in triplicate.

MTT assays. LN229 and U87 cells were plated in 96-well plates in medium containing $10 \%$ FBS at $\sim 3,000$ cells per well $24 \mathrm{~h}$ after transfected siRNA. For quantitation of cell viability, cultures were stained after 4 days in MTT assays. In brief, $20 \mu 1$ of $5 \mathrm{mg} / \mathrm{ml} \mathrm{MTT} \mathrm{(thiazolyl} \mathrm{blue)} \mathrm{solution}$ was added to each well and incubated for $4 \mathrm{~h}$ at $37^{\circ} \mathrm{C}$. The medium was removed from each well and the resulting MTT formazan was solubilized in $150 \mu \mathrm{l}$ of DMSO. Each solution was measured spectrophotometrically at $490 \mathrm{~nm}$.
Colony formation assay. LN229 and U87 cells were transfected with siRNA or a negative control for $48 \mathrm{~h}$ and then plated into six-orifice plate (1,000 per orifice) and transfected with siRNA one more time on day 6 . Until day 12 , plates were washed with PBS and stained with crystal violet. The number of colonies with $>30$ cells was counted. The colonies were manually counted using a microscope.

Western blot analysis. After cell treatment, cell lysates were prepared via lysis buffer, electrophoresed onto SDS-polyacrylamide gels and transferred to polyvinylidene difluoride membranes. Membranes were probed with rabbit antibodies against POSTN and glyceraldehyde-3-phosphate dehydrogenase (GAPDH) (A-3; Santa Cruz Biotechnology) at dilutions of 1:1,000. Blots were detected with horseradish peroxidase-labeled anti-rabbit antibodies (1:5,000 dilution), developed using enhanced chemiluminescence (ECL) reagents (Amersham Pharmacia, UK). The primary antibody against MMP-9 (Oncogene, Boston, MA), was used to examine protein expression.

Statistical analysis. Quantitative results represent mean \pm standard deviation. For the microarray data, if multiple probes were used for a single candidate gene, then the reference sequence of each probe was searched in GenBank and probes detecting exons were chosen for further analysis. Overall survival (OS) time was calculated from the date of diagnosis until death or the last follow-up contact. OS of the 
A

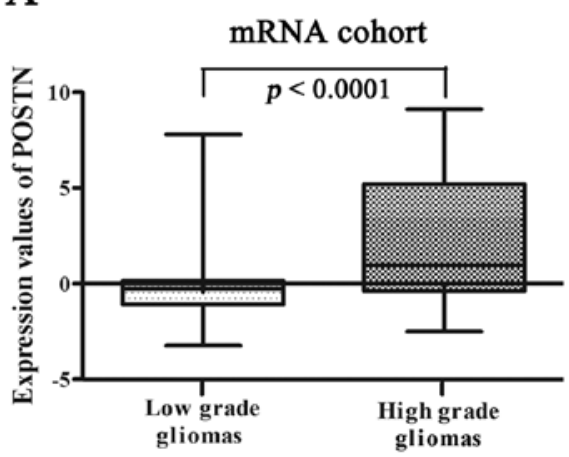

B

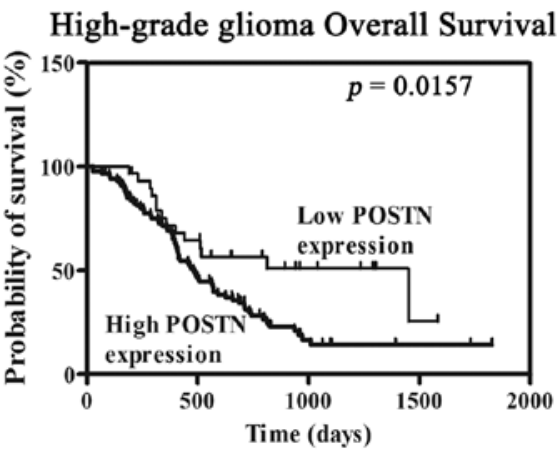

Figure 1. The expression of POSTN in gliomas and its association with survival in high-grade gliomas. (A) Low-grade gliomas and high-grade gliomas expression patterns of POSTN in the mRNA cohort containing 220 frozen glioma tissues. (B) Kaplan-Meier survival curves according to the expression of POSTN in 123 frozen high-grade glioma tissues. The log-rank test was used to calculate p-values. High expression $>$ median expression, low expression $\leq$ median expression.

Table III. POSTN expression is correlated with glioma progression.

\begin{tabular}{lcc} 
& \multicolumn{2}{c}{ Degree of POSTN immunoreactivity (\%) } \\
\cline { 2 - 3 } Characteristics & Low & High \\
\hline Low-grade $^{\mathrm{a}}$ & $13 / 24(54.2)$ & $11 / 24(45.8)$ \\
High-grade $^{\mathrm{a}}$ & $9 / 47(19.1)$ & $38 / 47(80.9)$ \\
III $^{\mathrm{b}}$ & $3 / 9(33.3)$ & $6 / 9(66.7)$ \\
IV $^{\mathrm{b}}$ & $6 / 38(15.8)$ & $32 / 38(84.2)$ \\
\hline
\end{tabular}

${ }^{\mathrm{a}} \mathrm{p}=0.006 ;{ }^{\mathrm{b}} \mathrm{p}=0.464$.

low- and high-gene expression groups were compared using the Kaplan-Meier plotting method and log-rank tests. For POSTN expression analyses using immunohistochemistry analysis in the CGGA cohort, the difference between high and low POSTN-expressing tissues was assessed using $\chi^{2}$ test. Gene ontology (GO) analysis was performed using DAVID (18). All analyses were two-tailed. p-values of $<0.05$ were considered statistically significant. Analyses were performed using Matlab 2009b, GraphPad Prism (GraphPad Software, La Jolla, CA) and SPSS version 13.0 (SPSS Inc., Chicago, IL).

Differences of tumor cell invasion and expression of POSTN and MMP-9 between treated and control groups were analyzed by t-test. Cox regression was used to correlate POSTN expression with PFS and OS of high-grade glioma patients. Factors with corresponding $\mathrm{p}$-values of $<0.1$ in the univariate analysis was applied for further multivariate Cox regression model.

\section{Results}

POSTN is relative to glioma grade progression and confers a poor prognosis of high-grade glioma patients based on large cohorts. The expression of POSTN was measured in a series of 220 glioma samples (97 low-grade gliomas, 34 anaplastic gliomas and 89 GBMs) via microarrays. POSTN was significantly upregulated as identified using significance analysis of microarrays (SAM). As shown in Fig. 1A, high-grade gliomas demonstrated a significant increase in POSTN transcript levels compared to the mean expression levels observed in low-grade gliomas $(\mathrm{p}<0.0001)$. The correlation between POSTN expression and overall survival was measured through Kaplan-Meier survival curve analysis with a log-rank comparison. POSTN expression was inversely correlated with overall survival in the high-grade glioma samples ( $\mathrm{p}=0.0157)$ (Fig. 1B).

POSTN is tightly associated with the biological processes of cell invasion and proliferation. A total of 3,677 probes significantly correlated $(\mathrm{R}>0.4$ or $<-0.4, \mathrm{p}<0.05)$ with POSTN expression levels (Fig. 2; 2,249 probes with positive correlation and 1,428 with negative correlation). Arrows indicate genes that are discussed in the text include MMP-11, MMP-2, MMP-25, MMP-14, MMP-9, MMP-7, MMP-1, MMP-19 and NFkB1. Gene ontology analysis (GO) showed that the POSTN positive correlative gene sets related to cell invasion and proliferation were significantly enriched in the cases with POSTN overexpression (Table I). Using a $\mathrm{p}<0.001,38$ biologic processes mainly related to cell migration, cell proliferation, cell motility and extracellular matrix organization were significantly enriched in the high-grade glioma samples with POSTN overexpression.

Validated patient demographics. Seventy-one mainland Han Chinese glioma patients who were receiving standard treatment were included in this study. The patient population consisted of 40 males and 31 females with a median age of 45 years (range 18-71). There were 24 patients with astrocytoma (A, WHO grade II), 9 with anaplastic astrocytoma (AA, WHO grade III) and 38 with glioblastoma multiforme (GBM; WHO grade IV). Of the 71 cases, 52 underwent total tumor resection and 19 underwent subtotal tumor resection. Preoperative KPS scores ranged from 50-100 (median 80). Fifty-six patients died after a median follow-up of 32.4 months (range 16-71 months). The median progression-free survival (PFS) was 406 days [95\% confidence interval (CI) (347-465 days] and the median overall survival (OS) was 501 days (95\% CI, 487-623 days).

Examination of POSTN expression level and its association with survival. Immunohistochemical staining showed POSTN 


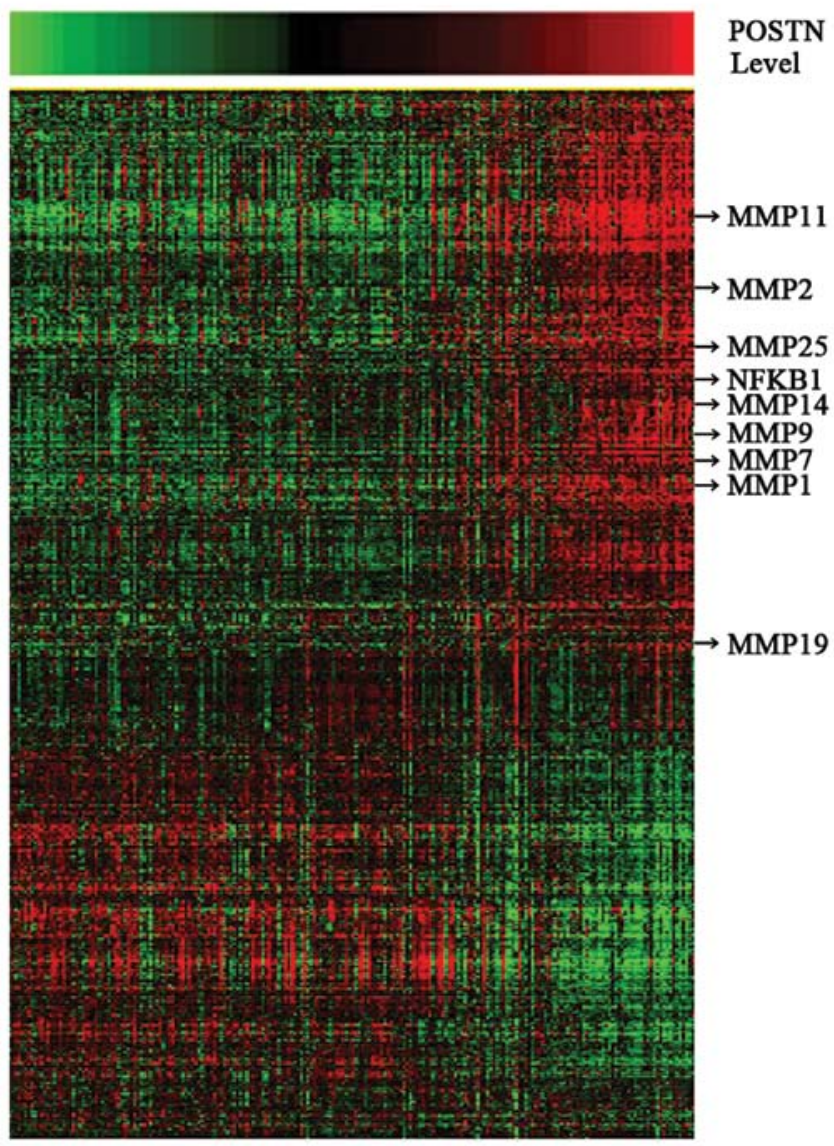

Figure 2. Heat map of the gene-expression signature correlated with POSTN expression. Rows represent probe sets and columns represent patients. Patients are from left to right in order of increasing POSTN expression. Expression levels of the probe sets are presented with green demonstrating expression less than and red expression greater than the median value for the given probe set. Arrows indicate genes that are discussed in the text.

expression ranged from low to high in WHO grades II, III and IV gliomas and was intensely stained (score >2) in $69.01 \%$ (49/71) of cases. POSTN was expressed in both tumor cells and stroma, but expression was higher in the stroma than in the cells (Fig. 3). We analyzed the correlation between POSTN protein expression and histological staging of gliomas. As summarized in Table III, POSTN expression was significantly higher in high-grade gliomas compared to low-grade gliomas $(\mathrm{p}<0.01)$, suggesting that POSTN expression level was associated with glioma progression. Survival analysis showed that patients with high POSTN expression had significant shorter overall survival (OS, $\mathrm{p}=0.032$ ) and progression-free survival (PFS, $\mathrm{p}=0.009$ ) than those with low expression respectively (Fig. 4).

High POSTN expression predicted poor prognosis in high-grade gliomas. In 47 high-grade glioma patients, immunohistochemical detection revealed high POSTN expression in tissue samples from 38 patients (80.9\%), while samples from nine patients (19.1\%) displayed low expression (Table III). POSTN expression was correlated with PFS and OS by univariate analysis $(\mathrm{p}=0.009$ and 0.032 , respectively) (Tables IV and V). Patients with high POSTN expression had median PFS and OS times of 382 and 460 days, respectively, compared to 683 and 1,099 days, respectively, in patients with low POSTN expression. Preoperative KPS score was also correlated with PFS $(\mathrm{p}=0.005)$ and OS $(\mathrm{p}=0.002)$. There were no significant associations between age, gender or extent of resection and PFS or OS (Tables IV and V). The multivariate Cox proportional hazards model, after adjusting for age, KPS score and extent of resection, identified high POSTN expression as an independent unfavorable prognostic factor for PFS [hazard ratio (HR), 3.762; $\mathrm{p}=0.009$ ] and OS (HR, 2.816; $\mathrm{p}=0.037$ ).

POSTN expression correlates with expression of matrix metalloproteinase-9 (MMP-9). Western blot analysis identified POSTN expression in LN229 and U87 human glioblastoma cell lines (Fig. 5A). A previous study showed that siRNA could effectively inhibit POSTN (19). The expression of POSTN in the control group was $\sim 6$-fold that in the siPOSTN (transfected siRNA) group in LN229 cells $(\mathrm{p}<0.001)$ and $\sim 2$-fold that in U87 cells ( $\mathrm{p}<0.001$ ). These results indicate that POSTN siRNA inhibited constitutively activated POSTN. To confirm the effect of POSTN on the regulation of intracellular molecules involved in aggressive cell behavior, we examined its influence on MMP-9 expression. High-POSTN-expressing cells expressed higher levels of MMP-9 compared to siPOSTN cells (Fig. 5A). MMP-9 expression in the control group was $\sim 5$-fold that in the siPOSTN group in LN229 cells $(\mathrm{p}<0.001)$ and $\sim 2$-fold that in $\mathrm{U} 87$ cells $(\mathrm{p}<0.001)$.

POSTN promotes invasion and proliferation of LN229 and U87 cell lines. To explore whether the cell invasion was
A

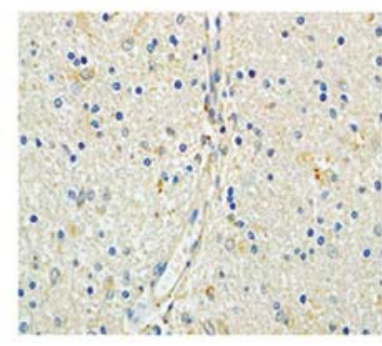

B

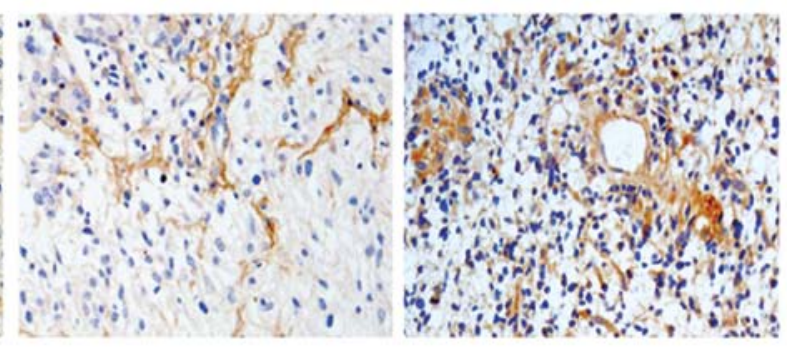

Figure 3. POSTN expression correlates with glioma progression and prognosis. Immunohistochemical staining showed POSTN expression ranged from low to high: astrocytoma (WHO grade II) (A), anaplastic astrocytoma (WHO grade III) (B) and glioblastoma (WHO grade IV) (C). POSTN was expressed in both tumor cells and stroma, but expression was higher in the stroma than in the cells. 
A

Validated cohort Progression-free Survival

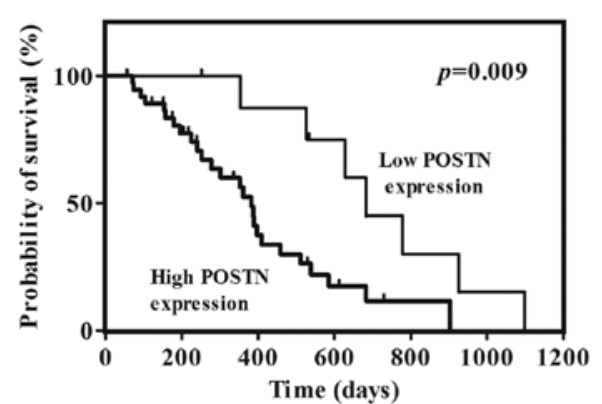

B

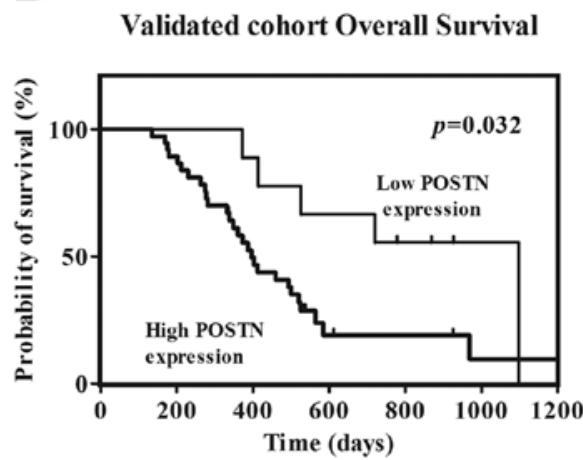

Figure 4. Kaplan-Meier survival estimates of validated cohort (71 independent glioma samples) progression-free survival (A) and overall survival (B) according to POSTN expression level in high-grade glioma patients. High expression, $\geq 2$; low expression, $<2$.

Table IV. Variables related to progression-free survival (PFS) in 47 high-grade glioma with combined treatment: univariate and multivariate analysis.

\begin{tabular}{|c|c|c|c|c|c|c|c|}
\hline \multirow[b]{2}{*}{ Variable } & \multirow[b]{2}{*}{$\begin{array}{c}\text { No. of } \\
\text { patients }\end{array}$} & \multicolumn{3}{|c|}{ Univariate analysis } & \multicolumn{3}{|c|}{ Multivariate analysis } \\
\hline & & $\begin{array}{c}\text { Median PFS } \\
\text { (days) }\end{array}$ & $\begin{array}{c}95 \% \text { CI } \\
\text { (days) }\end{array}$ & p-value & $\begin{array}{c}\text { Relative } \\
\text { risk }\end{array}$ & $95 \% \mathrm{CI}$ & p-value \\
\hline
\end{tabular}

\section{Gender}

Male

28

387

293-481

0.426

Female

19

396

205-587

Age (years)

$\leq 50$

25

388

321-455

$>50$

22

511

300-722

0.780

KPS

$<80$

15

277

320-334

$\geq 80$

32

511

339-683

0.005

0.262

0.105-0.656

0.004

Extent of resection

$\begin{array}{lllll}\text { Subtotal } & 19 & 361 & 188-534 & \\ \text { Total } & 28 & 511 & 278-744 & 0.202\end{array}$

POSTN-expression

\begin{tabular}{lrrrrrr} 
Low & 9 & 683 & $550-816$ & & & \\
High & 38 & 382 & $326-438$ & 0.009 & 3.762 & $1.390-10.186$ \\
\hline
\end{tabular}

dependent on POSTN activity, we measured cell invasion after POSTN knockdown in LN229 and U87 cell lines. In Fig. 5B, transwell invasion shows that the siRNA significantly attenuated the effect of POSTN on cell invasion $(\mathrm{p}<0.05)$. Colony formation assays showed that knockdown POSTN in LN229 and U87 cells led to an decrease in focus numbers $(\mathrm{p}<0.05$, Fig. 5C). Consistent with the colony formation assay results, MTT assay showed a significant decrease in proliferation was observed in siPOSTN LN229 and U87 cell lines compared to cells transfected with control (Fig. 5D), suggesting that POSTN enhances proliferation of LN229 and U87 cell lines.

\section{Discussion}

In the present study, we investigated the expression level of POSTN in two independent cohorts of almost 300 glioma patients in total. We further compared the expression levels of POSTN in low-grade (astrocytoma, oligodendrocytoma and oligoastrocytoma) and high-grade (anaplastic gliomas and glioblastomas) human gliomas and demonstrated a significant increase in POSTN expression from low- to highgrade tumors. POSTN was an independent prognostic factor predicting PFS and OS in high-grade gliomas, indicating a significant correlation between POSTN expression and clin- 
Table V. Variables related to OS in 47 high-grade glioma with combined treatment: univariate and multivariate analysis.

\begin{tabular}{|c|c|c|c|c|c|c|c|}
\hline \multirow[b]{2}{*}{ Variable } & \multirow[b]{2}{*}{$\begin{array}{l}\text { No. of } \\
\text { patients }\end{array}$} & \multicolumn{3}{|c|}{ Univariate analysis } & \multicolumn{3}{|c|}{ Multivariate analysis } \\
\hline & & $\begin{array}{l}\text { Median OS } \\
\quad \text { (days) }\end{array}$ & $\begin{array}{c}95 \% \mathrm{CI} \\
\text { (days) }\end{array}$ & p-value & $\begin{array}{l}\text { Relative } \\
\text { risk }\end{array}$ & $95 \% \mathrm{CI}$ & $\mathrm{p}$-value \\
\hline \multicolumn{8}{|l|}{ Gender } \\
\hline Male & 28 & 387 & $324-449$ & & & & \\
\hline Female & 19 & 525 & $399-651$ & 0.386 & & & \\
\hline \multicolumn{8}{|c|}{ Age (years) } \\
\hline$\leq 50$ & 25 & 501 & $367-635$ & & & & \\
\hline$>50$ & 22 & 396 & $290-502$ & 0.434 & & & \\
\hline \multicolumn{8}{|l|}{ KPS } \\
\hline$<80$ & 15 & 348 & $244-452$ & & & & \\
\hline$\geq 80$ & 32 & 525 & $405-645$ & 0.002 & 0.312 & $0.142-0.682$ & 0.004 \\
\hline \multicolumn{8}{|c|}{ Extent of resection } \\
\hline Subtotal & 19 & 412 & $206-618$ & & & & \\
\hline Total & 28 & 460 & $272-648$ & 0.214 & & & \\
\hline \multicolumn{8}{|c|}{ POSTN-expression } \\
\hline Low & 9 & 1099 & NR & & & & \\
\hline High & 38 & 396 & $339-453$ & 0.032 & 2.816 & $1.062-7.463$ & 0.037 \\
\hline
\end{tabular}

NR, not reached; CI, confidence interval; KPS, Karnofsky performance status.
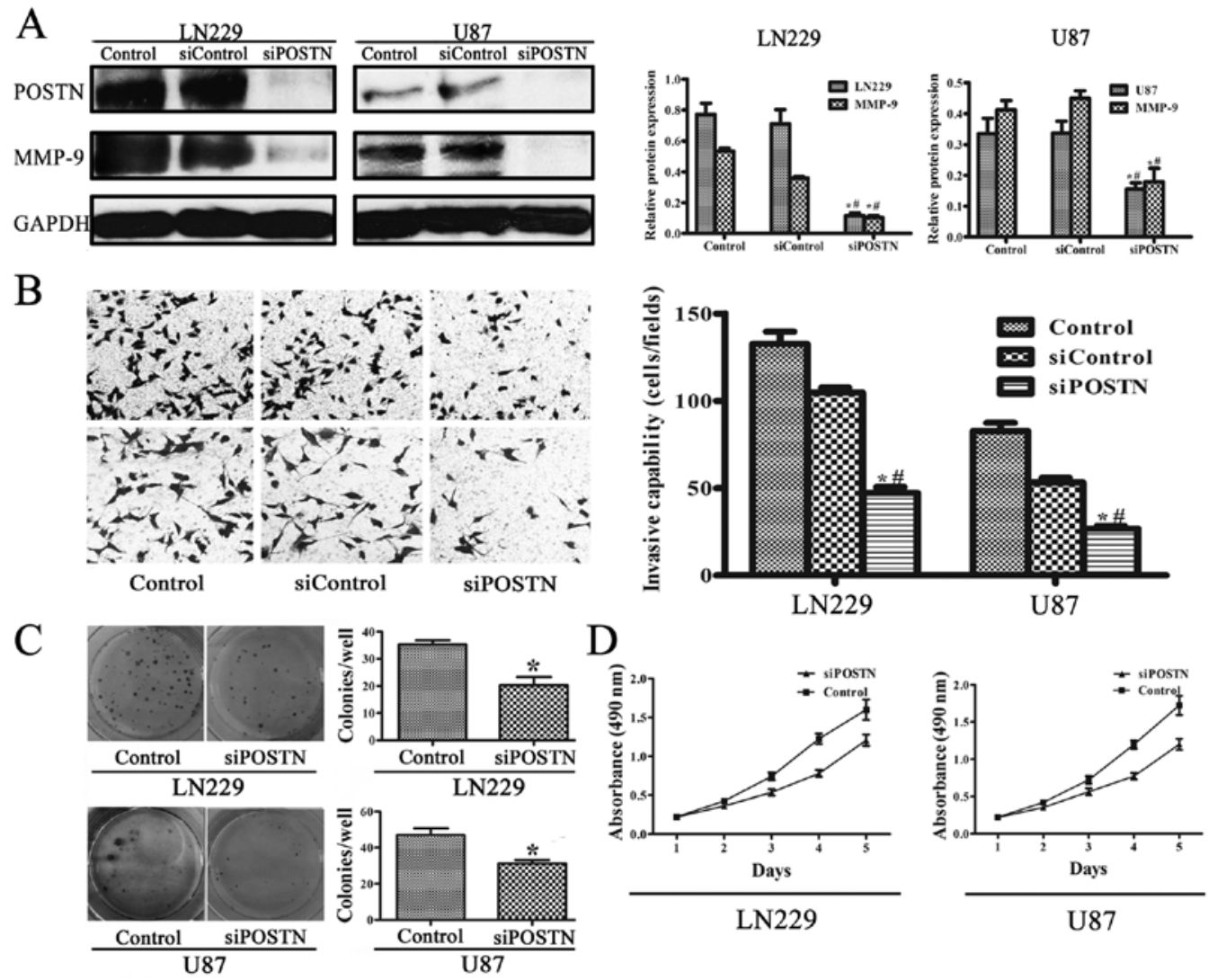

Figure 5. POSTN expression correlates with expression of matrix metalloproteinase-9 (MMP-9) and promotes invasion and proliferation of LN229 and U87 cell lines. (A) Expression of MMP-9 was significantly related to POSTN expression. MMP-9 expression was reduced in POSTN knockdown cells. Expression levels of POSTN and MMP-9 relative to GAPDH shown as a histogram. " $\mathrm{p}<0.05$ vs. control; ${ }^{*} \mathrm{p}<0.05$ vs. sicontrol. (B) Invasion of LN229 and U87 cell lines assessed by transwell assay. The number of invading cells was significantly less in the siPOSTN group compared to the control and siControl groups. Number of invading cells shown as a histogram. ${ }^{*} \mathrm{p}<0.05$ vs. control; ${ }^{*} \mathrm{p}<0.05$ vs. siControl. (C) The number of colonies formed by cells treated with siRNA of POSTN was decreased compared to control cells $(\mathrm{p}<0.05)$. (D) MTT assays were performed after knockdown POSTN. A decrease in absorbance was observed. 
ical outcome in glioma patients validated by 71 independent samples. POSTN functional analyses were performed in LN229 and U87 cell lines. MMP-9 was an effector of POSTN signaling in glioma cells. As far as we know, this is the first report on the expression difference, function and mechanism of POSTN in all grades of glioma, while previous reports only focus on glioblastoma (14-16).

Glioma is the most common and diffusely infiltrating brain tumor, with characteristic invasive patterns including preferential invasion along white-matter tracts, butterfly lesions after crossing the corpus callosum, perineuronal satellitosis, perivascular growth and subpial spread (20). The invasive characteristics of the most malignant glioma cells probably result from the activation of a genetic program controlling cell proliferation, remodeling of the extracellular matrix, cell invasion and migration and the formation of new blood vessels (21), allowing these cells to spread throughout the brain. This invasiveness of glioma cells is a major cause of therapeutic failure, especially in GBM $(2,22,23)$. Although most recurrences occur close to the tumor resection site, distant recurrences $>2 \mathrm{~cm}$ are also common $(24,25)$, indicating that the invasive phenotype is acquired early in tumorigenesis. Gliomas grow invasively and proliferate quickly, so clarification of the molecular mechanisms, especially those associated with cellular migration and invasion, is thus crucial to allow better predictions of glioma patient prognosis and response to therapies. POSTN has been found to increase aggressive cell behavior and exogenous expression of POSTN promoted cells to undergo epithelial-mesenchymal transition (19). Preventing glioma cells from invading adjacent brain parenchyma and more distant sites is thus a vital step in improving treatment.

Although POSTN expression has been detected in some cancers, it is not a general characteristic of tumors. For example, no POSTN expression was detected in cholangiocarcinoma cell lines, while there was high expression in fibroblasts (26). In contrast, POSTN was detected in cancer cells from head and neck, colon and ovary cancers and has been proposed to induce tumorigenic activity of cancer cells via an autocrine mechanism $(8,12)$. Another report showed that both stroma and tumor cells produced POSTN in melanoma, but POSTN was not expressed in blood cancers (27). Contie et al (4) confirmed that in addition to secretion, breast cancer cells also captured POSTN from the surrounding stroma. We detected POSTN expression in tumor cells and the stroma in gliomas (Fig. 3). It has been confirmed that fibroblasts can secrete POSTN $(3,26)$, but there are no fibroblasts in the brain and it is possible that glioma cells secrete POSTN via an autocrine mechanism, as confirmed by the results of western blotting in U87 and LN 229 glioma cell lines (Fig. 5A). POSTN expression in most parts of gliomas may account for their invasive growth pattern. In non-small cell lung cancer, POSTN was highly expressed only at the periphery of the tumor, with no expression within the tumor tissue (28). In contrast, POSTN is expressed at both the periphery and within gliomas and has been detected in both glioma cells and mesenchyme, as verified by glioma tissue immunohistochemical staining (Fig. 3). These results suggest that POSTN promotes the invasion of glioma cells and that these changes exist throughout the glioma tissue, in contrast to tumors with non-invasive growth patterns.
In the present study, two hundred and twenty samples with large variability in POSTN transcript levels were subjected to whole genome gene profiling. We observed a positive correlation of POSTN expression (Fig. 2) with a series of tumor genes on invasion and proliferation, including MMP superfamily and NFKB1 $(29,30)$. Gene ontology analysis (GO) showed also that the POSTN positive correlative gene sets related to cell migration and proliferation were significantly enriched in the cases with POSTN overexpression (Table I). MMP-9, a member of MMP superfamily, elevated expression and activity allow tumor cells to degrade and penetrate extracellular matrix $(31,32)$. MMP-9 is known to be an important factor in tumor invasion $(33,34)$ and in addition to the direct effects of POSTN on cell behavior, knockdown of POSTN was associated with a subsequent decrease in MMP-9 expression. These results suggest that POSTN expression may reflect the microenvironment within glioma tissues and we therefore conclude that POSTN promotes the invasive ability of glioma cells at least partly via MMP-9, highlighting MMP-9 as an effector of POSTN signaling in glioma cells (Fig. 5A).

In conclusion, our study demonstrated that POSTN expression correlated with glioma patient survival, as well as to glioma grade and is an independent prognostic factor in highgrade glioma patients. The results of this study demonstrate the important functional and molecular mechanisms of POSTN in tumor invasion and proliferation. POSTN promoted glioma cell invasiveness in vitro, accompanied by MMP-9 expression. The correlation between POSTN expression and increased glioma invasion and proliferation suggest that it could be used as a prognostic biomarker for poor outcome and it may also represent a future therapeutic target.

\section{Acknowledgements}

We would like to thank Dr Susan Furness for her critical reading and Professor Chen (Beijing Sanbo Brain Hospital) for IHC technical support. This study was supported by grants National Key Project of Science and Technology Supporting Programs of China (no. 2007BAI05B08) and National High Technology Research and Development Program (2012AA02A508). Additional support was provided through grant International Science and Technology Cooperation Program (2012DFA30470).

\section{References}

1. Zhang J, Sarkar S and Yong VW: The chemokine stromal cell derived factor-1 (CXCL12) promotes glioma invasiveness through MT2-matrix metalloproteinase. Carcinogenesis 26: 2069-2077, 2005.

2. Wang Y, Chen L, Bao Z, et al: Inhibition of STAT3 reverses alkylator resistance through modulation of the AKT and betacatenin signaling pathways. Oncol Rep 26: 1173-1180, 2011.

3. Malanchi I, Santamaria-Martinez A, Susanto E, et al: Interactions between cancer stem cells and their niche govern metastatic colonization. Nature 481: 85-89, 2012.

4. Contie S, Voorzanger-Rousselot N, Litvin J, Clezardin P and Garnero P: Increased expression and serum levels of the stromal cell-secreted protein periostin in breast cancer bone metastases. Int J Cancer 128: 352-360, 2011.

5. Sasaki H, Yu CY, Dai M, et al: Elevated serum periostin levels in patients with bone metastases from breast but not lung cancer. Breast Cancer Res Treat 77: 245-252, 2003

6. Ben QW, Jin XL, Liu J, Cai X, Yuan F and Yuan YZ: Periostin, a matrix specific protein, is associated with proliferation and invasion of pancreatic cancer. Oncol Rep 25: 709-716, 2011. 
7. Ruan K, Bao S and Ouyang G: The multifaceted role of periostin in tumorigenesis. Cell Mol Life Sci 66: 2219-2230, 2009.

8. Kudo Y, Ogawa I, Kitajima S, et al: Periostin promotes invasion and anchorage-independent growth in the metastatic process of head and neck cancer. Cancer Res 66: 6928-6935, 2006.

9. Siriwardena BS, Kudo Y, Ogawa I, et al: Periostin is frequently overexpressed and enhances invasion and angiogenesis in oral cancer. Br J Cancer 95: 1396-1403, 2006.

10. Erkan M, Kleeff J, Gorbachevski A, et al: Periostin creates a tumor-supportive microenvironment in the pancreas by sustaining fibrogenic stellate cell activity. Gastroenterology 132: 1447-1464, 2007.

11. Tai IT, Dai M and Chen LB: Periostin induction in tumor cell line explants and inhibition of in vitro cell growth by anti-periostin antibodies. Carcinogenesis 26: 908-915, 2005.

12. Gillan L, Matei D, Fishman DA, Gerbin CS, Karlan BY and Chang DD: Periostin secreted by epithelial ovarian carcinoma is a ligand for alpha(V)beta(3) and alpha(V)beta(5) integrins and promotes cell motility. Cancer Res 62: 5358-5364, 2002.

13. Shao R, Bao S, Bai X, et al: Acquired expression of periostin by human breast cancers promotes tumor angiogenesis through up-regulation of vascular endothelial growth factor receptor 2 expression. Mol Cell Biol 24: 3992-4003, 2004.

14. Zinn PO, Mahajan B, Sathyan P, et al: Radiogenomic mapping of edema/cellular invasion MRI-phenotypes in glioblastoma multiforme. PLoS One 6: e25451, 2011.

15. Gottlieb A, Varshavsky R, Linial M and Horn D: UFFizi: a generic platform for ranking informative features. BMC Bioinformatics 11: 300, 2010.

16. Mikheeva SA, Mikheev AM, Petit A, et al: TWIST1 promotes invasion through mesenchymal change in human glioblastoma. Mol Cancer 9: 194, 2010.

17. Tusher VG, Tibshirani R and Chu G: Significance analysis of microarrays applied to the ionizing radiation response. Proc Natl Acad Sci USA 98: 5116-5121, 2001.

18. Huang da W, Sherman BT and Lempicki RA: Systematic and integrative analysis of large gene lists using DAVID bioinformatics resources. Nat Protoc 4: 44-57, 2009.

19. Yan $W$ and Shao R: Transduction of a mesenchyme-specific gene periostin into $293 \mathrm{~T}$ cells induces cell invasive activity through epithelial-mesenchymal transformation. J Biol Chem 281: 19700-19708, 2006.

20. Claes A, Idema AJ and Wesseling P: Diffuse glioma growth: a guerilla war. Acta Neuropathol 114: 443-458, 2007.

21. Dauer DJ, Ferraro B, Song L, et al: Stat 3 regulates genes common to both wound healing and cancer. Oncogene 24: 3397-3408, 2005.

22. Senft C, Priester M, Polacin M, et al: Inhibition of the JAK-2/ STAT3 signaling pathway impedes the migratory and invasive potential of human glioblastoma cells. J Neurooncol 101: 393-403, 2011.
23. Stupp R, Mason WP, van den Bent MJ, et al: Radiotherapy plus concomitant and adjuvant temozolomide for glioblastoma. N Engl J Med 352: 987-996, 2005.

24. Giese A, Bjerkvig R, Berens ME and Westphal M: Cost of migration: invasion of malignant gliomas and implications for treatment. J Clin Oncol 21: 1624-1636, 2003.

25. Wick W, Stupp R, Beule AC, et al: A novel tool to analyze MRI recurrence patterns in glioblastoma. Neurooncology 10: 1019-1024, 2008.

26. Utispan $\mathrm{K}$, Thuwajit $\mathrm{P}$, Abiko $\mathrm{Y}$, et al: Gene expression profiling of cholangiocarcinoma-derived fibroblast reveals alterations related to tumor progression and indicates periostin as a poor prognostic marker. Mol Cancer 9: 13, 2010.

27. Tilman G, Mattiussi M, Brasseur F, van Baren N and Decottignies A: Human periostin gene expression in normal tissues, tumors and melanoma: evidences for periostin production by both stromal and melanoma cells. Mol Cancer 6: 80, 2007.

28. Sasaki H, Dai M, Auclair D, et al: Serum level of the periostin, a homologue of an insect cell adhesion molecule, as a prognostic marker in nonsmall cell lung carcinomas. Cancer 92: 843-848, 2001.

29. Park MH and Min do S: Quercetin-induced downregulation of phospholipase D1 inhibits proliferation and invasion in U87 glioma cells. Biochem Biophys Res Commun 412: 710-715, 2011.

30. Mut M, Amos S and Hussaini IM: PKC alpha phosphorylates cytosolic NF-kappaB/p65 and PKC delta delays nuclear translocation of NF-kappaB/p65 in U1242 glioblastoma cells. Turk Neurosurg 20: 277-285, 2010

31. Lakka SS, Gondi CS, Yanamandra N, et al: Inhibition of cathepsin B and MMP-9 gene expression in glioblastoma cell line via RNA interference reduces tumor cell invasion, tumor growth and angiogenesis. Oncogene 23: 4681-4689, 2004.

32. Kupferman ME, Fini ME, Muller WJ, Weber R, Cheng Y and Muschel RJ: Matrix metalloproteinase 9 promoter activity is induced coincident with invasion during tumor progression. Am J Pathol 157: 1777-1783, 2000.

33. Kang CS, Pu PY, Li YH, et al: An in vitro study on the suppressive effect of glioma cell growth induced by plasmid-based small interference RNA (siRNA) targeting human epidermal growth factor receptor. J Neurooncol 74: 267-273, 2005.

34. Friedberg MH, Glantz MJ, Klempner MS, Cole BF and Perides G: Specific matrix metalloproteinase profiles in the cerebrospinal fluid correlated with the presence of malignant astrocytomas, brain metastases and carcinomatous meningitis. Cancer 82 : 923-930, 1998 . 